\title{
EDUCATIONAL LEVEL AND GENDER-AGE CHARACTERISTICS OF THE EPIDEMIOLOGY OF HOSPITAL ANXIETY IN SURGICAL PATIENTS
}

\author{
MD, Gaisa T. Kairov' ${ }^{1}$ PhD, Alexander G. Martusevich ${ }^{2}$, Danila S. Zhulin ${ }^{3}$, Denis Y. Chernov ${ }^{3}$, \\ Daria V. Potapova ${ }^{4}$ \\ ${ }^{1}$ Department of Anaesthesiology and Reanimatology, Siberian State Medical University, Tomsk, Russia; \\ ${ }^{2}$ Department of General Surgery, Siberian State Medical University, Tomsk, Russia; \\ ${ }^{3} 5$ th year student of the General Medicine Faculty, Siberian State Medical University, Tomsk, Russia; \\ ${ }^{4} 4$ th year student of the Pediatric Faculty, Siberian State Medical University, Tomsk, Russia
}

\section{DOI: https://doi.org/10.31435/rsglobal_conf/30112020/7271}

\begin{abstract}
A prospective cohort study was performed in 138 patients aged 48-85 years (68 men and 70 women, mean age $42.0 \pm 18.5$ years) who were to undergo surgery for diseases of the abdominal cavity. Patients aged 18-33 and 34-49 years are most often subject to an average level of preoperative anxiety, which dictates the need to include adequate doses of tranquilizers in premedication in the preoperative period. Patients in the age range of 50-65 years and 66-81years most often suffer from high preoperative anxiety, which requires special attention of the anesthesiologist-resuscitator and the appointment of adequate doses of anxiolytic drugs in preoperative premedication, especially in female patients. Taking into account the individual psychological characteristics of patients before surgery will help to qualitatively protect the patient from preoperative stress, comfortably carry out surgery, and reduce the frequency of intra- and postoperative complications associated with inadequate premedication and patient preparation for surgery.
\end{abstract}

Keywords: HADS scale, surgical patients, educational level, gender, age

Introduction. The scale of the prevalence of depression in the world is evidenced by data from the World Health Organization (2017), which reported 300 million cases of depression, of which $77 \%$ of respondents associate this psychophysiological disorder with pain syndrome [1]. It is now generally accepted that the patient's preoperative anxiety is not only a risk factor for the development of postoperative pain syndrome, but in many cases affects the reparative processes after surgery [2-5].

In particular, the results of a Norwegian cross-sectional population study, in which 5731 respondents (men and women) took part in two age ranges: 46-49 years old and 70-74 years old, showed that age, education and income, physical activity, smoking, alcohol consumption and a healthy diet are inversely related to the incidence of clinical occurrence of hospital depression [6]. Studies by Periañez et al (2020) showed the presence of preoperative anxiety in $47.7 \%$ of patients, and depression was detected in $13.8 \%$ of cases. According to the authors, only patients with preoperative anxiety experienced postoperative pain of varying intensity in almost half of the cases, which gave researchers reason to consider depression as a predictor of the development of postoperative pain syndrome, which is due to uncertainty about improving health after surgery and fears about the success of surgery [7].

Along with this, according to systematic reviews and meta-analyzes, it was found that depression is an independent risk factor for the development of chronic non-communicable diseases [8-12]. Thus, the results of a meta-analysis of prospective studies presented by Engum (2007), Rotella and Mannucci (2013) indicate that under conditions of depression, the chance of developing type 2 diabetes mellitus (DM) increases by $60 \%[10,13]$ and the risk of clinical realization of cardiovascular diseases increases [10-12].

However, in 50-80\% of cases these neurotic and anxious - phobic disorders are not detected by outpatient doctors, and at the hospital stage of treatment in the preoperative period they are not recognized in $70 \%$ of cases, which increases the degree of operational anesthetic risk. complicates the work of anesthesiologists and surgeons at all stages of treatment [14]. Unfortunately, doctors at the hospital stage do not pay due attention to this aspect of the disease, which (in turn) leads to the appointment of preoperative premedication without taking into account the individual psychological characteristics of the patient. According to Stepanova et al (2015), in the routine clinical practice of an anesthesiologist, the psychological portrait of the patient's personality is rarely taken into account when choosing an analgesic strategy at all stages of surgical treatment, including the postoperative period [15] 
In the context of the above difficulties and problems, the purpose of our study was to study the gender and age characteristics of the epidemiology of hospital anxiety in patients with a surgical profile.

Material and research methods. A prospective cohort study was performed in 138 patients aged 48-85 years (68 men and 70 women, mean age $42.0 \pm 18.5$ years) who were to undergo surgery for diseases of the abdominal cavity.

Study design. Inclusion criteria: age not less than 48 years old and not more than 85 years old; the absence of mental illness in the anamnesis and in the immediate family; not taking psychotropic drugs six months before enrollment in the study; no indication of participation in other clinical trials at the time of inclusion in this study; informed consent to participate in this clinical trial. Exclusion criteria: age less than 48 years old and more than 85 years old; a history of mental illness and in the immediate family; an indication of taking psychotropic drugs six months before inclusion in the study; indication of participation in other clinical trials; lack of informed consent to participate in this clinical trial.

Research methods. Psychological diagnostics of the level of anxiety was carried out at the preoperative stage using the hospital scale of anxiety and depression (HADS), which is validated in Russia. The questionnaire includes 14 questions to identify and assess the severity of depression and anxiety in a hospital setting over the past 2 weeks. There are 3 areas of values: normal: $0-7$ points, no reliably pronounced symptoms of anxiety and depression; subclinically severe anxiety/depression: 810 points; clinically severe anxiety/depression - 10 points or more. The scale allows you to separately assess the presence of signs of anxiety and depression [16].

In this work, we focused on the indicator of anxiety as a more common clinical phenomenon at the preoperative stage.

Statistical Analyses. Statistical processing of the research results was carried out in the environment of the "Statistica 8.0" package. The normal distribution of indicators was checked using the Shapiro-Wilk test (W-test). The Mann-Whitney test (U-test) was used to assess the significance of differences between the samples. Differences were considered significant at a significance level of $\mathrm{p}<.05$ [17].

Research results. The analysis of age characteristics of the frequency of distribution of levels of hospital anxiety showed that a low level of anxiety in the age group of 18-33 years was detected in $13.5 \%$ of cases, at the age of 34-49 years - in 15.5\% of the examined, in patients aged 50-65 years - only $2.5 \%$ of respondents and at the age of $66-81-$ in $6.5 \%$ of patients. The average level of anxiety in the age group of 18-33 years was recorded in 54\% of the surveyed, at the age of $34-49$ years - in $47 \%$ of patients, in patients aged 50-65 years, the average level of hospital anxiety was found in $36 \%$ of the respondents and at the age of $66-81$ years - in $26.5 \%$ of patients. A high level of anxiety in the age group 18-33 years was revealed in $32.5 \%$ of patients, at the age of $34-49$ years - in $37.5 \%$ of cases, at the age of 50-65 years in $61.5 \%$ of respondents and in the age group 66-81 years old, a high level of preoperative anxiety was recorded in $67 \%$ of the examined.

An assessment of the gender characteristics of the epidemiology of preoperative hospital anxiety, performed in 68 men and 70 women, showed that a low level of anxiety was found in $14 \%$ of male patients and in $4 \%$ of women. There were no significant differences in the frequency of distribution of the average level of anxiety in the surveyed men and women in our study. In particular, the average level of anxiety was recorded in $46 \%$ of cases in men and in $45 \%$ of women surveyed. A high level of preoperative anxiety was recorded in $40 \%$ of male patients and in $51 \%$ of cases in the examined women.

Analysis of the influence of education level on the frequency and severity of hospital anxiety showed that patients with secondary education had a low level of anxiety in $12 \%$ of cases, an average level of anxiety was recorded in 53\% of cases, and a high level of anxiety was found in 35\% of patients. In those surveyed with secondary specialized education, only in 5\% of cases a low level of anxiety was recorded. The average level of hospital anxiety in patients with secondary education was found in $25 \%$ of the examined and in $70 \%$ of patients a high level of anxiety was revealed. According to our data, patients with higher education are characterized by medium and high levels of anxiety, recorded in $44 \%$ and $45 \%$, respectively. At the same time, only $11 \%$ of patients with higher education showed a low level of hospital anxiety.

Discussion of research results. In our opinion, the reason for the revealed gender differences in the frequency and severity of preoperative anxiety is associated with the peculiarities of the mentality of women, who (in contrast to men) are characterized by a negative self-assessment of 
health [18] and a lower level of emotion control [19], which (in turn) determines their frequent adherence to manifestations of depression, fear and anxiety [20].

Our results of assessing the frequency and severity of hospital anxiety in patients with different levels of education contradict the studies of Feinstein et al. (2006) and Mackenbach et al. (2015), according to which the level of education is a determining factor in lifestyle, medical culture and culture of health, reflects higher life expectancy and lower mortality rates, in comparison with the same indicators of persons with a different educational qualification [21]. Further research is needed to determine the reasons for this contradiction. The high frequency of severe anxiety, revealed in our study in patients of the older age group, is consistent with the opinion of Polishchuk et al. (2008), according to which the main reasons for the high frequency of anxiety, anxiety-phobic and anxiety-hypochondriacal personality disorders are associated with awareness of one's condition, the presence of various comorbidities and a lack of confidence in a favorable outcome of pain relief and surgery [22].

Conclusions. Patients aged 18-33 and 34-49 years are most often subject to an average level of pre-operative anxiety, which dictates the need to include adequate doses of tranquilizers in premedication in the preoperative period. Patients in the age range of 50-65 years and 66-81 years most often suffer from high preoperative anxiety, which requires special attention of the anesthesiologist-resuscitator and the appointment of adequate doses of anxiolytic drugs in preoperative premedication, especially in female patients.

Taking into account the individual psychological characteristics of patients before surgery will help to qualitatively protect the patient from preoperative stress, comfortably carry out surgery, and reduce the frequency of intra- and postoperative complications associated with inadequate premedication and patient preparation for surgery.

\section{REFERENCES}

1. WHO. 2017. Retrieved June 13, 2017 from the World Health Organization http://www.who.int/mediacentre/factsheets/fs369/en/ American Pain Foundation. (2006). Voices of chronic pain survey. Retrieved from http://www.painmed.org/patientcenter/facts_on_pain.aspx\#refer.

2. Janssen K.J., Moons K.G., Kalkman C.J., Grobbee D.E., Vergouwe Y. Updating methods im-proved the performance of a clinical prediction model in new patients. J. Clin. Epidemiol. 2008; 61: 76-86.

3. Munafo M.R., Stevenson J. Anxiety and surgical recovery. Reinterpreting the literature. J. Psy-chosom. Res. 2001; 51: 589-96.

4. Rotboll-Nielsen P., Rudin A., Werner M.U. Prediction of postoperative pain. Curr Anaesth. Crit. Care. 2007; 18: 157-65

5. Marcoci D., Brain B., Ciurea M., Parvaneseu H., Vrabete M. Psychological status, real pain and antioxidant capacity of plasma, could make the prediction about the post-trauma wound healing. Rom. J. Morphol. Embryol. 2011; 52(1): 459-63.

6. Jacka FN, Mykletun A., Berk M. et al. The association between habitual diet quality and the common mental disorders in community-dwelling adults:The Hordaland Health Study. J Psychosom. Med. 2011; 73 (6): 483-90.

7. Periañez CAH, Diaz MAC, Bonisson PLV, Simino GPR, Barbosa MH, De Mattia AL. Relationship of anxiety and preoperative depression with post-operative pain. Texto Contexto Enferm [Internet]. 2020 [cited YEAR MONTH DAY]; 29:e20180499. Available from: http://dx.doi.org/10.1590/1980-265X-TCE-2018-0499.

8. Katon W.J. Epidemiology and treatment of depression in patients with chronic medical illness. Dia-logues Clin. Neurosci. 2011;13 (1): 7-23.

9. Atlantis E., Fahey P., Cochrane B., Smith S. Bidirectional associations between clinically rele-vant depression or anxiety and chronic obstructive pulmonary disease (COPD): a systematic review and metaanalysis. Chest 2013;144: 766-77.

10. Rotella F., Mannucci E. Depression as a risk factor for diabetes: a meta-analysis of longitudinal studies. J Clin Psychiatry. 2013;74 (1):31-37.

11. Rugulies R. Depression as a predictor for coronary heart disease. A review and meta-analysis. Am J Prev Med 2002;23 (1): 51-61.

12. Patten S.B., Williams J.V., Lavorato D.H. et al. Major depression as a risk factor for high blood pressure: epidemiologic evidence from a national longitudinal study. J Psychosom Med 2009;71 (3): 273-279.

13. Engum A. The role of depression and anxiety in onset of diabetes in a large population-based study. J Psychosom Res 2007; 62 (1): 31-8.

14. Gilbody S. M. (2003). Improving the detection and management of depression in primary care. Quality and Safety in Health Care.2003;12 (2):149-155. doi:10.1136/qhc.12.2.149.

15. Stepanova Y.V., Mazurok V.A., Shchelkova O.Y. Psychophysiological aspects of pain percep-tion in early postoperative period. Regional Anesthesia and Acute Pain Management.2015; 9 (2):9-13 (in Russian). 
16. Andryushenko A.V., Drobizhev M.Yu., Dobrovolskiy A.V. Comparative assessment of the CES-D, BDI and HADS (d) scales in the diagnosis of depression in general medical practice. J. Neurol. Psychiatry. 2003; 5: 11-7 (in Russian).

17. Figurnov V.E. Data analysis on a computer. M: Finance and statistics; 1995 (in Russian).

18. Kayumova M.M., Akimov A.M., Gorbunova T.Yu., Gafarov V.V. Self-assessment of the health status of men and women in an open population of a medium-urbanized city of Western Siberia: gender characteristics. Siberian scientific medical journal. 2019; 39 (5): 149-155. doi: 10.15372/SSMJ20190518 (in Russian).

19. Antropova L.K., Kulikov V.Yu., Osintseva A.A. Gender features of emotional intelligence in individuals with different lateral profile. Journal of Siberian Medical Sciences.2015; 5: 49.

20. Andreeva I.N. Gender differences in the expression of the components of emotional intelli-gence. Psychological journal. 2008; 1:39-43 (in Russian)

21. Feinstein, L., Sabates, R., Anderson, T. M., Sorhaindo, A., \& Hammod, C. What are the effects of education on health? Measuring the effects of education on health and civic engagement: Proceedings of the Copenhagen Symposium, OECD. 2006:173-354.

22. Polishchuk Yu.I., Letnikova Z.V. Anxiety and anxiety-phobic neurotic disorders at a later age. Clinical gerontology. 2008; 8: 46-50 (in Russian). 